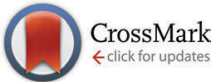

Cite this: Chem. Commun., 2015, 51, 11382

Received 24th April 2015,

Accepted 4th June 2015

DOI: $10.1039 / c 5 c c 03408 f$

www.rsc.org/chemcomm

\section{An asymmetric BODIPY triad with panchromatic absorption for high-performance red-edge laser emission $\dagger$}

\author{
Gonzalo Duran-Sampedro, ${ }^{a}$ Antonia R. Agarrabeitia, ${ }^{a}$ Inmaculada Garcia-Moreno, ${ }^{b}$ \\ Leire Gartzia-Rivero, ${ }^{C}$ Santiago de la Moya, ${ }^{a}$ Jorge Bañuelos, ${ }^{* c}$ \\ Íñigo López-Arbeloa ${ }^{c}$ and María J. Ortiz ${ }^{{ }^{a}}$
}

\begin{abstract}
A rational design of an unprecedented asymmetric cassette triad based entirely on BODIPY chromophores allows efficient light harvesting over the UV-vis spectral region, leading to a bright and stable red-edge laser emission via efficient energy-transfer processes.
\end{abstract}

Long wavelength (>600 nm) fluorescent dyes have found applications in a wide variety of fields, such as laser printing, information storage, displays and solar power conversion. ${ }^{1}$ They were also proved to be useful tools in biomedical applications (including photodynamic therapies), ${ }^{2}$ due mainly to the deeper penetration into tissues of this kind of light. ${ }^{3}$ Although a number of longwavelength emitting commercial dyes with reasonable efficiency are available, ${ }^{4}$ these dyes have important drawbacks: low absorption at the standard pump wavelengths (355 and $532 \mathrm{~nm}$ ) and/or poor photostability. One approach to overcome these drawbacks is the straightforward synthesis of energy transfer cassettes, ${ }^{5}$ which has proved to be a powerful strategy in fields such as lightharvesting systems and sensors. ${ }^{6}$ Thus, these complex molecular multichromophoric systems offer two main valuable advantages with regard to simpler systems and physical mixtures of individual (non-covalently linked) chromophores: (1) the possibility of excellent excitation energy transfer (EET) efficiencies upon absorption over a broad spectral window, to yield almost exclusively the emission of a target chromophore unit; (2) the possibility of performing the excitation far away from the emission region, which enhances both the system photostability and the sensibility when detecting the emission signal. However, this approach has not

\footnotetext{
${ }^{a}$ Depto. de Química Orgánica I, Facultad de CC. Químicas, Universidad Complutense de Madrid, Ciudad Universitaria s/n, 28040, Madrid, Spain. E-mail:mjortiz@ucm.es

${ }^{b}$ Depto. de Sistemas de Baja Dimensionalidad, Superficies y Materia Condensada, Instituto de Química-Física "Rocasolano", C.S.I.C., Serrano 119, 28006 Madrid, Spain

${ }^{c}$ Depto. de Química Física, Universidad del Pais Vasco-EHU, Apartado 644, 48080, Bilbao, Spain. E-mail: jorge.banuelos@ehu.es

$\dagger$ Electronic supplementary information (ESI) available: Experimental, photophysical and computational details, characterization data and NMR spectra of the new compounds, as well as Fig. S1-S4 and Table S1. See DOI: 10.1039/c5cc03408f
}

been tried to date to develop panchromatic dyes with enhanced red-edge laser emission.

In this regard, the rational design of a multichromophoric molecular system with the aforementioned EET benefits is challenging, since the effectiveness of each individual EET event depends on the mutual separation and relative orientation of the involved donor and acceptor moieties. BODIPYs (4,4-difluoro-4-bora-3a,4adiaza-s-indacenes) were demonstrated to be valuable scaffolds to design cascade-like EET architectures, ${ }^{7}$ since they can be properly functionalized to assure strong absorption throughout the visible spectrum. ${ }^{8}$ On the other hand, high efficiency of monochromatic emission beyond $650 \mathrm{~nm}$ can be obtained from certain convenientlyfunctionalized BODIPYs. ${ }^{9}$ On the basis of this synthetic versatility of the BODIPY chromophores for designing energy-transfer systems, herein we report a straightforward, efficient and cost-effective synthetic protocol to produce an unprecedented panchromatic all-BODIPY cassette triad (1 in Fig. 1) enabling highly efficient and stable laser emission at $665 \mathrm{~nm}$.

The asymmetric molecular architecture of $\mathbf{1}$ is not trivial, as it was judiciously designed to get the desired photophysical properties through a simpler synthetic route. Thus, its twisted architecture was selected to avoid supramolecular aggregation by $\pi-\pi$ stacking interactions, which are known to have a deleterious effect on laser action. However, compared to symmetric BODIPY systems, which can be straightforwardly synthesized (e.g., from

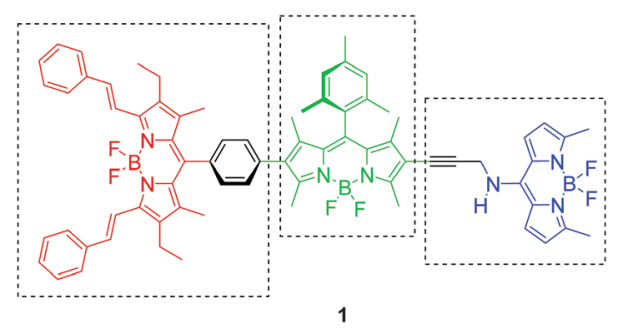

Fig. 1 Developed cassette triad. Key BODIPY moieties in different colours (spacers in black). Individual key synthetic building-block fragments inside dotted windows. 
pyrrole derivatives), the facile and scalable synthesis of low symmetric BODIPY analogues is still challenging.

The lateral 8-aminoBODIPY moiety of $\mathbf{1}$ (the blue fragment in Fig. 1) was selected as the short-wavelength donor due to the known strong absorption and highly efficient and stable emission in the blue-green spectral region exhibited by related individual BODIPYs $(400-500 \mathrm{~nm}) .{ }^{10}$ On the other hand, the central 8-mesitylBODIPY moiety (the green moiety in Fig. 1) was chosen on the basis of its expected strong absorption and emission in the green-orange spectral region (490-600 nm), ${ }^{11}$ which should allow the required dual behaviour of the central core inside the triad (donor and acceptor moieties), but also because the corresponding isolated BODIPY gives a convenient synthetic building block to let its lateral asymmetric functionalization occur as shown later. Finally, the 3,5-distyrylBODIPY chromophore (the red fragment in Fig. 1) was selected due to its likely strong absorption and high efficient emission in the red-edge spectral region (600-750 nm). ${ }^{9}$ Moreover, the molecular assembly of the said moieties was achieved through relatively short and rigid spacers (twisted 1,4-phenylene $\$$ and 1,3-propynylidene) to get the desired photophysical properties. Thus, the phenylene bridge is crucial to maintain the twisted architecture of the spacer, avoiding its aggregation. Besides, this twisted spacer should maintain the corresponding linked BODIPYs electronically isolated. Alternatively, the non- $\pi$-conjugated propynylidene spacer is key to keep the "blue" identity of the lateral 8-aminoBODIPY, maintaining the linked chromophores isolated. The selection of the spacers takes into account the synthetic factors also, since they must allow the straightforward assembly of synthetically-accessible BODIPY-based building blocks, as mentioned below.

Thus, triad 1 was attained by the straightforward convergent synthetic route shown in Fig. 2, which was judiciously designed on the basis of using workable palladium-catalysed carbon-carbon coupling reactions (Sonogashira and Suzuki). Synthetically-accessible 2,6-diiodoBODIPY 3 (easily obtainable from accessible 2 ) $^{12}$ is the key intermediate of the designed route, since it could be subjected to controlled (stepped) carbon-carbon coupling reaction towards the final desired triad. Thus, the Sonogashira coupling of 3 with the blue $N$-propargyl-8-aminoBODIPY 5 (obtained from commercial $4^{13}$ by using the methodology described by Peña et al. ${ }^{10}$ ) allowed the easy preparation of diad 6 (Fig. 2). On the other hand, subjecting synthetically accessible $7^{14}$ to Knoevenagel-like reaction with benzaldehyde $/ \mathrm{K}_{2} \mathrm{CO}_{3}$ (standard red-shifting distyryl functionalization of 3,5-dimethylBODIPYs ${ }^{9}$ ) allows obtaining boronicester-based red BODIPY 8, which could be straightforwardly coupled to diad 6 by Suzuki reaction, to afford desired 1 (Fig. 2).

As a consequence of the mentioned rational design, the absorption spectrum of triad 1 (Fig. 3) resulted to be the sum of the bands of the involved individual chromophores. Accordingly, four strong absorption bands at $631,536,426$ and $342 \mathrm{~nm}\left(\varepsilon_{\max }\right.$ up to $10^{5} \mathrm{M}^{-1} \mathrm{~cm}^{-1}$, see Table S1 in the ESI $\dagger$ ) covering the whole near-ultraviolet-to-red spectral region were recorded. The three vis bands were assigned to the corresponding absorption of the electronically-isolated BODIPY chromophores of $\mathbf{1}$, by comparing the absorption spectrum of $\mathbf{1}$ with those individually obtained from parent dyes $\mathbf{2 , 5}$ and 8 (Fig. 3). The observed red shift of the band at $536 \mathrm{~nm}$, when compared with that obtained from 2 , is mainly due to the conjugation of

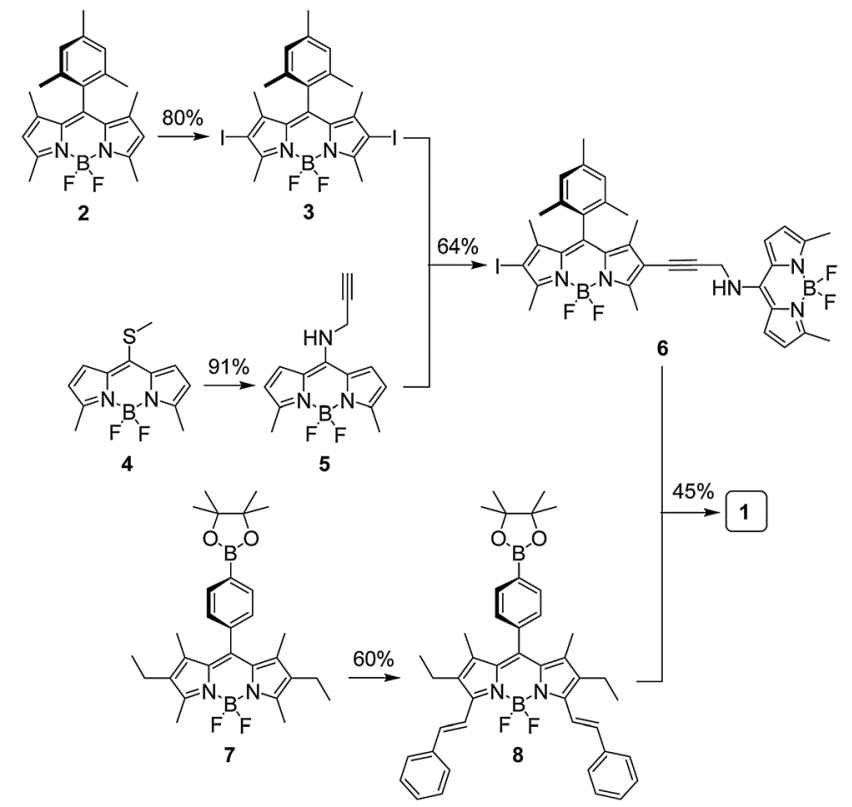

Fig. 2 Convergent synthetic route to 1 (yields over arrows; see ESI† for details).

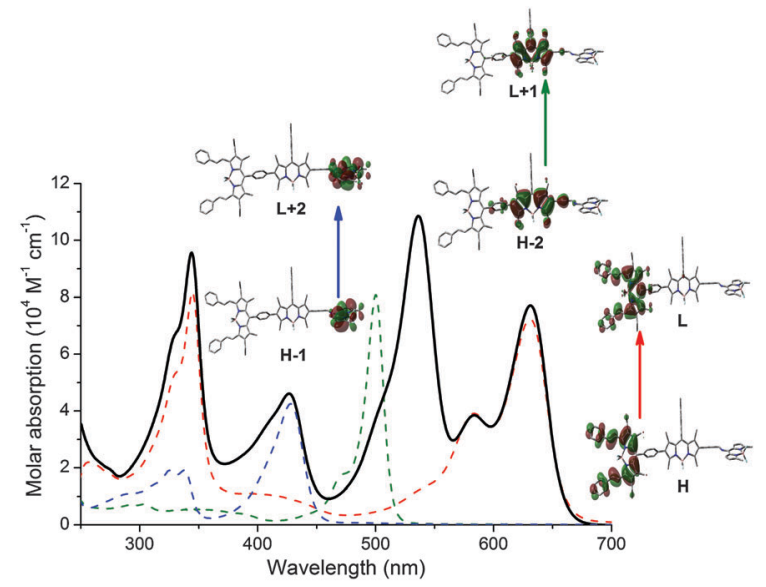

Fig. 3 Absorption spectra of $\mathbf{1}$ (solid line), $\mathbf{2}$ (green), $\mathbf{5}$ (blue) and $\mathbf{8}$ (red) in diluted ethyl acetate, as well as computed molecular orbitals involved in each visible transition of $\mathbf{1}$. For better visualization of the contour maps, see Fig. S1 in the ESI.†

the central BODIPY core of $\mathbf{1}$ with the propynylidene rest (see Fig. 3). On the other hand, the ultraviolet (UV) band at $342 \mathrm{~nm}$ results from the summed UV absorptions of the involved individual BODIPY chromophores, being the distyryl chromophore the main contributor (see Fig. 3). Theoretical calculations conducted on 1 (B3LYP/6-31 $+\mathrm{g}^{*}$, see ESI $\dagger$ ) also support the claimed electronic isolation of chromophores, by predicting the involvement of molecular orbitals placed exclusively at each BODIPY fragment for the vis electronic transitions associated with each vis absorption band, (see Fig. 3). Therefore, the cassette-required selective excitation of chromophores should be possible in $\mathbf{1}$.

Indeed, the selective excitation of each chromophore in $\mathbf{1}$ leads to a strong red fluorescence from the distyryl fragment 


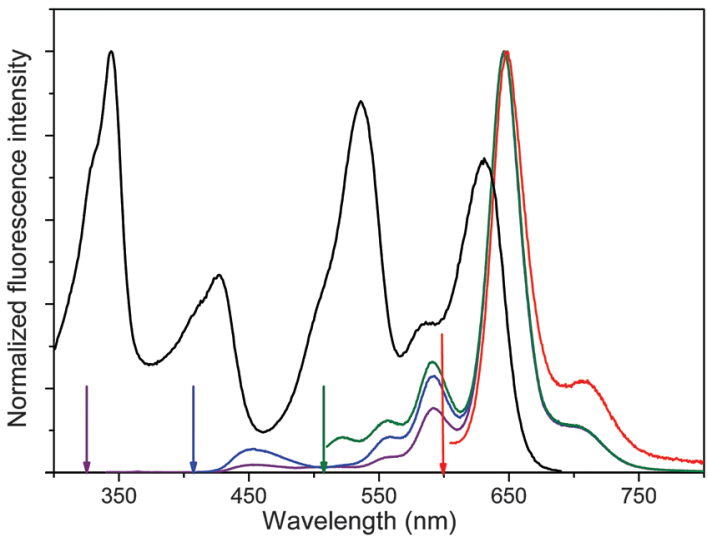

Fig. 4 Fluorescence spectra of 1 at different excitation wavelengths along the UV-vis (indicated by arrows), and the excitation spectrum monitored at $700 \mathrm{~nm}$ (black) in diluted solutions (ethyl acetate).

(645 $\mathrm{nm}, \phi=72 \%$; see Table S1 in ESI $\dagger$ ), regardless of the excitation wavelength along the whole UV-vis spectrum (Fig. 4). Therefore, efficient EET processes take place, as demonstrated also by the excitation spectra monitored at a long-enough wavelength (see Fig. 4), which matches with the absorption spectrum showing its characteristic four bands ( $c f$., Fig. 3 and 4).

The efficiencies of the EET processes occurring in $\mathbf{1}$ can be estimated from the quenching of the emission of the donor chromophores, lateral blue and central green, induced by the presence of the corresponding acceptor chromophores, central green and lateral red (see Fig. S2 and Table S1 in the ESI $\dagger$ ). Thus, the efficiency of the EET from the blue donor to the green acceptor resulted to be $99 \%$, whereas the efficiency from the green donor to the final red acceptor was $91 \%$. It must be noted here that such extremely efficient EET in diluted solution could not be achieved from the physical mixture of the individual components of the triad, even at high concentrations (see Fig. S3 in the ESI $\dagger$ ), which shows the advantages of using covalent linkages to impose appropriate short distances and relative dispositions between chromophores, to enable efficient EET processes. ${ }^{5 d}$ The intramolecular EETs between chromophores should obey a Förster-resonance energy-transfer (FRET) mechanism, being extremely efficient due to the involved short separation distances and the notable spectral overlaps (especially high for the blue and green chromophores; see Fig. S4 in the ESI $\dagger$ ). Besides, a contribution of the through-bond energy transfer (TBET) mechanism (not allowed in mixtures of individual dyes) should also be feasible in the hop from the central green chromophore to the red one, since the involved phenylene spacer could enable the required electronic connection through its $\pi$-conjugated orbitals. In fact, the calculated efficiency for this EET process is quite high, despite the lower spectral overlap existing for the green and red chromophores (see Fig. S4 in the ESI $\dagger$ ). In contrast, such a mechanism is less feasible in the hop from the lateral blue chromophore to the central one, owing to the involved methylene-bridge of the involved propynylidene spacer, which should reduce the electron-exchange probability.

The optimized photophysics of $\mathbf{1}$, obtained from its carefully designed structure, results in enhanced laser behaviour as a laser dye when compared to those obtained individually from the parent monochromophoric dyes. Thus, this singular structure enables 1 to lase efficiently in the red spectral region under pumping either in the UV (355 nm) or in the vis (532 nm) spectral regions, due to the mentioned cassette behaviour, but also because the covalent binding of its three BODIPY chromophores leads to a significant increase in the absorptions, at both pumping wavelengths, when compared to those exhibited individually by the related monochromophoric parent dyes. This is a key factor from the point of view of the laser action, since it allows reducing significantly the required gain-media concentrations, avoiding consequently dye-solubility problems, or dye quenching and/or aggregation processes, all of them with detrimental effects on laser emission. For instance, the new triad exhibits molar absorption coefficients of $9.5 \times 10^{4}$ and $11 \times 10^{4} \mathrm{M}^{-1} \mathrm{~cm}^{-1}$ at 355 and $532 \mathrm{~nm}$, respectively, which are above those exhibited individually by the comparison parent dyes (see Fig. 3).

Under transversal pump conditions at $355 \mathrm{~nm}$, broad-linewidth laser emission, with pump threshold energy of $\sim 0.6 \mathrm{~mJ}$, divergence of $5 \mathrm{mrad}$ and pulse duration of $8 \mathrm{~ns}$ full width at half maximum (FWHM), was obtained from a triad solution placed in a simple plane-plane non-tunable resonator (see ESI $\dagger$ for experimental details). In agreement with the photophysical behaviour, the cascade of EET processes inside triad 1 leads to a highly efficient (up to $49 \%$ ) and monochromatic laser emission centered at $665 \mathrm{~nm}$. This laser efficiency is much higher than that recorded from parent red BODIPY 8, whose laser efficiency does not exceed the $30 \%$ value when pumped under identical experimental conditions, either at $355 \mathrm{~nm}$ or at $532 \mathrm{~nm}$. These results could be related to the following facts: (a) the lower absorption of 8 at $532 \mathrm{~nm}$, when compared to triad 1, making it necessary to increase significantly the dye concentration in the gain media to achieve the optimal pumping conditions, and (b) the energetic difference between absorbed (355 $\mathrm{nm}$ ) and emitted (665 nm) photons, which makes the emission process to be energetically more favourable when taking place through an EET cascade, as occurring in $\mathbf{1 .}$

Triad 1 also exhibits excellent photostability, maintaining $100 \%$ of the initial emission intensity after 100000 pump pulses (see ESI $\dagger$ for experimental details). Furthermore, the photostability of $\mathbf{1}$ was demonstrated to be noticeably higher than those exhibited individually by the corresponding triad components. As an example, under identical pumping conditions, the key individual blue and red components of $\mathbf{1}$ (i.e., the initial absorber and the final emiter; see $\mathbf{5}$ and $\mathbf{8}$ in Fig. 2) lose completely their laser emission after 100000 pump pulses. The same takes place when the three individual components are physically mixed in a single solution, which clearly demonstrates the benefits of embedding them into a proper single molecular architecture. In order to put this result in a proper perspective, the lasing parameters of the commercial BODIPY dye PM650, lasing at the same wavelength obtained from 1, were measured under identical experimental conditions. Noticeably and different from 1, PM650 could not be pumped at $355 \mathrm{~nm}$, since its low absorption at this wavelength makes necessary dye concentrations upcoming the dye solubility limit. On the other hand, pumping PM650 $\left(2 \times 10^{-3} \mathrm{M}\right.$ in ethyl acetate $)$ at 
$532 \mathrm{~nm}$ makes this dye to lase at $657 \mathrm{~nm}$ with $35 \%$ efficiency and $38 \%$ loss of the initial laser-induced fluorescence intensity after 100000 pump pulses. Therefore, triad 1 outperforms the lasing properties of PM650, especially taking into account that PM650 was only pumped at the longer $532 \mathrm{~nm}$ wavelength i.e., with less energetic photons minimizing the energy released into the medium and, therefore, reducing the rate and extension of the photodegradation process occurring when pumping with high-energy photons. Moreover, compared to PM650, triad 1 exhibits another important advantage, namely its high chemical stability in solution. Thus, PM650 has been reported to be highly unstable in certain solvents (most of them characterized as electron donors), ${ }^{15}$ becoming completely bleached after some hours (or days in the best case), and implying that its solutions have to be used and characterized just after preparation.

In conclusion, we have developed a straightforward and costeffective synthetic approach to attain an asymmetric all-BODIPY triad with panchromatic absorption and, simultaneously, efficient and photostable laser emission in the red-edge spectral region. The rational design of this molecular system ameliorates three key optical factors to improve its photonic applicability: four strong absorption bands with extinction absorption coefficients up to $10^{5} \mathrm{M}^{-1} \mathrm{~cm}^{-1}$, EET efficiency as high as $99 \%$, and laser efficiency up to $49 \%$, the latter being much higher than those exhibited by the parent red BODIPY mimicking the triad red chromophore, and by the commercial dye PM650, which is considered the bench-mark of the red-emitting BODIPY-based laser dyes. Further work is in progress to study the laser performance of the reported cassette, and future related ones, in solid media (e.g., in thin film by dispersing the molecular cassette in an appropriate polymer).

Financial support from the MINECO (MAT2014-51937-C3-1-P, -2-P and -3-P) of Spain, UCM (GR3/14-910107 and -910150) and Gobierno Vasco (IT339-10) is gratefully acknowledged. L.G.-R. thanks UPV-EHU for a postdoctoral fellowship and G.D.-S thanks MINECO for predoctoral fellowships.

\section{Notes and references}

\$ Neighbouring methyl groups make the 1,4-phenylene spacer of triad 1 to be twisted, as it occurs in the phenyl group of related monochromophoric BODIPYs. This effect is also shown in the computed triad structure (see Fig. 3 and Fig. S1 in the ESI $\dagger$ ).

1 (a) T. A. Fayed, in Reviews in Fluorescence, ed. C. D. Geddes, Springer, New York, 2011; (b) Tunable Laser Applications, ed. F. J. Duarte, CRC, New York, 2nd edn, 2009; (c) L. Bonardi, H. Kanaan, F. Camerel, P. Jolinat, P. Retailleau and R. Ziessel, Adv. Funct. Mater., 2008, 18, 401; (d) O. A. Bozdemir, S. Erbas-Cakmak, O. O. Ekiz, A. Dana and E. U. Akkaya, Angew. Chem., Int. Ed., 2011, 50, 10907.
2 (a) T. Yogo, Y. Urano, Y. Ishitsuka, F. Maniwa and T. Nagano, J. Am. Chem. Soc., 2005, 127, 12162; (b) T. Myochin, K. Hanaoka, T. Komatsu, T. Terai and T. Nagano, J. Am. Chem. Soc., 2012, 134, 13730.

3 R. Steiner, in Applied Laser Medicine, ed. H. P. Berlien and G. H. Müller, Sringer-Verlag, 2003.

4 Lambdachrome Laser Dyes, ed. U. Backmann, Lambda Physik, 2000.

5 (a) D. McQuade, A. Pullen and T. Swager, Chem. Rev., 2000, 100, 2537; (b) A. Coskun and E. U. Akkaya, J. Am. Chem. Soc., 2005, 127, 10464; (c) X. Zhang, Y. Xiao and X. Qian, Org. Lett., 2008, 10, 29; (d) Y. Xiao, D. Zhang, X. Qian, A. Costela, I. Garcia-Moreno, V. Martin, M. E. Perez-Ojeda, J. Bañuelos, L. Gartzia and I. Lopez Arbeloa, Chem. Commun., 2011, 47, 11513; (e) L. Cerdan, E. Enciso, V. Martin, J. Bañuelos, I. Lopez Arbeloa, A. Costela and I. GarciaMoreno, Nat. Photonics, 2012, 6, 621; $(f)$ J. Fan, M. Hu, P. Zhan and X. Peng, Chem. Soc. Rev., 2013, 42, 29.

6 (a) J. Warnan, F. Buchet, Y. Pellegrin, E. Blart and F. Odobel, Org. Lett., 2011, 13, 3944; (b) Y. Shi, R. B. M. Hill, J.-H. Yum, A. Duale, S. Barlow, M. Grätzel, S. R. Marder and M. K. Nazeeruddin, Angew. Chem., Int. Ed., 2011, 50, 6619; (c) N. C. Jeong, H.-J. Son, C. Prasittichai, C. Y. Lee, R. A. Jensen, O. K. Farha and J. T. Hupp, J. Am. Chem. Soc., 2012, 134, 19820; (d) D. K. Panda, F. S. Goodson, S. Ray and S. Saha, Chem. Commun., 2014, 50, 5358.

7 (a) J. Han, J. Jose, E. Mei and K. Burgess, Angew. Chem., Int. Ed., 2007, 46, 1684; (b) W. Lin, L. Yuan, Z. Cao, Y. Feng and J. Song, Angew. Chem., Int. Ed., 2010, 49, 375; (c) R. Ziessel, G. Ulrich, J. H. Oliver, T. Bura and A. Sutter, Chem. Commun., 2010, 46, 7978; (d) Y. Ueno, J. Jose, A. Loudet, C. Perez-Bolivar, P. Anzenbacher and K. Burgess, J. Am. Chem. Soc., 2011, 133, 51; (e) Y. Zhao, Y. Zhang, X. Lv, Y. Liu, M. Chen, P. Wang, J. Liu and W. Guo, J. Mater. Chem., 2011, 21, 13168; $(f)$ Q. Huaulmé, E. Cece, A. Mirlouop and R. Ziessel, Tetrahedron Lett., 2014, 55, 4953.

8 (a) S. Erten-Ela, M. D. Yilmaz, B. Icli, Y. Dede, S. Icli and E. U. Akkaya, Org. Lett., 2008, 10, 3299; (b) K. Umezawa, Y. Nakamura, H. Makino, D. Citterio and K. Suzuki, J. Am. Chem. Soc., 2008, 130, 1550; $(c)$ A. Haefele, C. Zedde, P. Retailleau, G. Ulrich and R. Ziessel, Org. Lett., 2008, 12, 1672; (d) S. Kolemen, O. A. Bozdemir, Y. Cakmak, G. Barin, S. Erten-Ela, M. Marszalek, Y.-H. Yum, S. M. Zakeeruddin, M. K. Nazeeruddin, M. Grátzel and E. U. Akkaya, Chem. Sci., 2011, 2, 949; (e) S. Zhu, J. Zhang, G. K. Vegesna, R. Pandey, F.-T. Luo, S. A. Green and H. Liu, Chem. Commun., 2011, 47, 3508; $(f)$ C. Qin, A. Mirloup, N. Leclerc, A. Islam, A. El-Shafei, L. Han and R. Ziessel, Adv. Energy Mater., 2014, 4, 85; (g) S. Kuhri, V. Engelhardt, R. Faust and D. M. Guldi, Chem. Sci., $2014,5,2580$.

9 H. Lu, J. Mack, Y. Yang and Z. Shen, Chem. Soc. Rev., 2014, 43, 4778. 10 (a) C. F. A. Gomez-Duran, I. Garcia-Moreno, A. Costela, V. Martin, R. Sastre, J. Bañuelos, F. Lopez Arbeloa, I. Lopez Arbeloa and E. Peña Cabrera, Chem. Commun., 2010, 46, 5103; (b) J. Bañuelos, V. Martin, C. F. A. Gomez-Duran, I. J. Arroyo Cordoba, E. Peña-Cabrera, I. Garcia-Moreno, A. Costela, M. E. Perez-Ojeda, T. Arbeloa and I. Lopez Arbeloa, Chem. - Eur. J., 2011, 17, 7261.

11 (a) A. Loudet and K. Burgess, Chem. Rev., 2007, 107, 4891; (b) G. Ulrich, R. Ziessel and A. Harriman, Angew. Chem., Int. Ed., $2008,47,1184$.

12 L. Fu, F.-L. Jian, D. Fortin, P. D. Harvey and Y. Liu, Chem. Commun., 2011, 46, 5503.

13 Purchased from Cuántico de México.

14 M. Koepf, A. Trabolsi, M. Elhabiri, J. A. Wytko, D. Paul, A. M. Albrecht-Gary and J. Weiss, Org. Lett., 2005, 7, 1279.

15 J. Bañuelos, T. Arbeloa, M. Liras, V. Martinez and F. Lopez Arbeloa, J. Photochem. Photobiol., A, 2006, 184, 298. 